IJMMS 28:7 (2001) 375-394

PII. S0161171201011450

http://ijmms.hindawi.com

(c) Hindawi Publishing Corp.

\title{
ON AN INFINITE SEQUENCE OF INVARIANT MEASURES FOR THE CUBIC NONLINEAR SCHRÖDINGER EQUATION
}

\author{
PETER E. ZHIDKOV
}

(Received 21 January 2001 and in revised form 28 May 2001)

\begin{abstract}
We consider the Cauchy problem periodic in the spatial variable for the usual cubic nonlinear Schrödinger equation and construct an infinite sequence of invariant measures associated with higher conservation laws for dynamical systems generated by this problem on appropriate phase spaces. In addition, we obtain sufficient conditions for the boundedness of the measures constructed.
\end{abstract}

2000 Mathematics Subject Classification. 35Q55, 37K99, 46N20.

1. Introduction. Consider the Cauchy problem periodic in the spatial variable for the cubic nonlinear Schrödinger equation

$$
\begin{gathered}
i \psi_{t}=-\psi_{x x}+2 \kappa|\psi|^{2} \psi, \quad x, t \in \mathbb{R}, \\
\psi(x+A, t)=\psi(x, t), \\
\psi\left(x, t_{0}\right)=\psi_{0}(x) .
\end{gathered}
$$

Here $i$ is the imaginary unit, $t_{0} \in \mathbb{R}, \psi=\psi(x, t)$ is an unknown complex function, $\psi_{0}$ is a complex function periodic in $x$ with the period $A>0$ and $\kappa=1$ or $\kappa=-1$. As it is well known, (1.1) supplied with condition (1.2) is formally a completely integrable Hamiltonian system possessing an infinite series of conservation laws $Q_{n}(\psi)$ which are real functionals quadratic with respect to the highest derivatives of the function $\psi$ formally satisfying the property $d / d t Q_{n}(\psi(\cdot, t))=0, n=0,1,2, \ldots$ (see Section 2 ). Further, it is known that in the finite-dimensional case to any conservation law $Q(p, q)$ of a Hamiltonian system of the kind

$$
\frac{d p(t)}{d t}=\nabla_{q} H(p, q), \quad \frac{d q(t)}{d t}=-\nabla_{p} H(p, q),
$$

where $H(p, q)$ is a smooth real function, $n$ is a natural number, and $p(\cdot), q(\cdot) \in \mathbb{R}^{n}$, there corresponds a family of invariant measures (IMs) with densities $f(Q(p, q)$ ) where $f(\cdot)$ is an arbitrary smooth real function. The problem we are interested in is whether this property is kept for the infinite-dimensional problem (1.1), (1.2), and (1.3), that is, whether conservation laws $Q_{n}(\psi), n \geq 2$, generate IMs, too.

Some recent papers are devoted to constructing IMs for dynamical systems (DS's) generated by nonlinear evolution partial differential equations of mathematical physics such as a nonlinear wave equation or a nonlinear Schrödinger equation (NLS) (cf. $[1,2,5,6,11,16,18,24,25,26,27,28,29]$ ). Formally, the early paper in this direction [1] does not contain the proof of the invariance of the measure considered in 
it. However, the invariance easily follows from the results presented in this paper. An IM for a physical system is considered in [6]. In [11], an IM is presented for a problem periodic in the spatial variable for the nonlinear wave equation

$$
u_{t t}-u_{x x}+u^{3}=0
$$

Unfortunately, some details of the proof seem to be not completely satisfactory in this paper. In [24], a result on IMs is presented for an NLS with a weak nonlinearity. Paper [26] contains an extension of this result to a nonlinear wave equation. In [27], a construction of an IM is presented for an abstract DS such that a lot of "soliton" equations are reducible to that form. Simultaneously, a result for a nonlinear wave equation similar to the above-described was proved in [18]. In [2], the results from [27] are reconstructed; the author established more careful proofs of results from [27].

All the above-indicated papers dealt with an NLS or a nonlinear wave equation (except [11]) contain results on IMs under severe constraints for nonlinearities. So, the problem appeared how to extend this approach onto a wider class of nonlinearities. Treatments of this problem are made in [5, 16, 25, 28, 29]. In [5], Bourgain, using his result from [3] on the existence of $L_{2}$-solutions for the Cauchy problem periodic in the spatial variable for a one-dimensional NLS with the power nonlinearity $\kappa|\psi|^{p-1} \psi$, constructed a bounded IM for $\kappa=-1$ and $1<p<5$. Similar results are contained in [16, 25, 28] (with $1<p<3$ if $\kappa=-1$ and $1<p<\infty$ if $\kappa=1$ in [25]) but in these papers suitable results on the well-posedness of initial-boundary problems for equations under consideration are not proved and are contained as hypotheses. In further publications Bourgain [5] constructed also IMs for a multidimensional NLS and, in [5], considered an IM for the Korteweg-de Vries equation.

Papers [1, 2, 5, 6, 11, 16, 18, 24, 25, 26, 27, 28] (except [18]) concern only with an IM associated with the concrete conservation law, the energy. At the same time, now it is known that certain evolution equations such as the Korteweg-de Vries equation or the cubic NLS possess countable sets of conservation laws and are formally infinitedimensional Hamiltonian systems. Therefore, since in the finite-dimensional case any conservation law of a Hamiltonian system leads to a family of corresponding IMs, the question naturally arises whether to higher conservation laws there correspond IMs in some infinite-dimensional cases. In [9], this question is considered for a discrete system of Moser-Calogero particles. A construction of an IM corresponding to a higher conservation law for the sinh-Gordon equation is contained in [18]. In [29], it is proved for the Cauchy problem periodic in the spatial variable for the Korteweg-de Vries equation that to any known conservation law of the kind

$$
L_{n}(u)=\int_{0}^{A}\left\{\frac{1}{2}\left(u_{x}^{(n)}\right)^{2}+q_{n}\left(u, \ldots, u_{x}^{(n-1)}\right)\right\} d x
$$

where $n \geq 3$ is an integer, there corresponds an IM for a DS generated by this equation. In the present paper, we continue the investigations began in [29] and present an infinite series of IMs for the problem (1.1), (1.2), and (1.3). This result is also reestablished (without proofs) in [30].

Now, we want to mention some applications of IMs. First, in $[1,6,7,8,15,16,18]$ they are used for constructing statistical mechanics of systems described by certain 
nonlinear partial differential equations (in [7, 8, 15], the problem of the invariance is not considered). Second, at the time when "soliton" equations began to be intensively studied, there arose a question known as the Fermi-Pasta-Ulam phenomenon (see [20]) which, roughly speaking, consists in the stability according to Poisson of all trajectories of a DS generated by a "soliton" equation. If one has a bounded IM for that DS (so that the measure of the whole phase space is finite), then the Poincaré recurrence theorem gives a (partial) explanation of this phenomenon. In this connection, it is important to note that, due to the problem (1.1), (1.2), and (1.3) being completely integrable, the method of the inverse scattering problem may give a more complete information about the recurrence and other properties of solutions. For example, such investigations have been performed by McKean and Trubowitz [17] for $C^{\infty}$-solutions and Bourgain [4] for $L_{2}$-solutions periodic in the spatial variable of the Korteweg-de Vries equation. In these papers, the almost periodicity of solutions is proved. At the same time, the author of the present paper does not know rigorous mathematical results implying the $X_{n}$-recurrence of solutions of the problem (1.1), (1.2), and (1.3) given by Theorem 2.5 .

2. Notation, preliminaries, and main results. In what follows, by $C, C_{1}, C_{2}, C^{\prime}$, $C^{\prime \prime}, \ldots$ we denote positive constants. Everywhere $t$ and $x$ are real variables. We fix a positive integer $n$ and $A>0$. Let $L_{2}$ be the usual Lebesgue space consisting of complex functions $u(x)$ of the argument $x$ periodic with the period $A$, equipped by the scalar product

$$
(f, g)=\int_{0}^{A} f(x) \overline{g(x)} d x
$$

and the norm $\|f\|=(f, f)^{1 / 2}$. Let $C^{\infty}$ be the set of complex functions $u(x)$ periodic with the period $A$ and infinitely differentiable. Let $\Delta$ be the closure in the space $L_{2}$ of the operator $-d^{2} / d x^{2}$ defined first on the set $C^{\infty}$. It is well known that $\Delta$ is a nonnegative selfadjoint operator in the space $L_{2}$. Further, let $n$ be a nonnegative integer and let $H^{n}$ be the usual Sobolev space which is the completion of the space $C^{\infty}$ taken with the scalar product

$$
(u, v)_{n}=(u, v)+\left(\frac{d^{n} u}{d x^{n}}, \frac{d^{n} v}{d x^{n}}\right)
$$

and the norm $\|u\|_{n}=(u, u)_{n}^{1 / 2}$. In fact, $L_{2}=H^{0}$ and $H^{n}$ are Hilbert spaces for $n=$ $0,1,2, \ldots$. Let $D_{x}^{n}=d^{n} / d x^{n}$ or $D_{x}^{n}=\partial^{n} / \partial x^{n}$ and $D_{x}=D_{x}^{1}$. Finally, consider a Banach space $X$ with a norm $\|\cdot\|_{X}$ and let $I$ be a connected subset of the real line $\mathbb{R}$. Then, by $C(I ; X)$ we denote the Banach space consisting of bounded continuous functions from $I$ into $X$, with the norm $\|u(t)\|_{C(I ; X)}=\sup _{t \in I}\|u(t)\|_{X}$.

Now we briefly recall basic facts from the theory of Gaussian measures in Hilbert spaces (for details, see $[10,21]$ ). Let $H$ be a separable real Hilbert space with a scalar product $(\cdot, \cdot)_{H}$ and let $S$ be a selfadjoint positive operator of trace class in the space $H$. Then, by definition, there exists an orthonormal basis $\left\{e_{k}\right\}_{k=1,2,3, \ldots}$ in the space $H$ consisting of eigenfunctions of this operator with the corresponding eigenvalues $\omega_{k}>0$ where $\omega_{k} \rightarrow+0$ as $k \rightarrow \infty$ and, in addition, $\sum_{k=1}^{\infty} \omega_{k}<\infty$. We call a set $M \subset H$ 
the cylindrical set if and only if

$$
M=\left\{f \in H \mid\left[\left(f, e_{1}\right)_{H}, \ldots,\left(f, e_{k}\right)_{H}\right] \in F\right\}
$$

for some integer positive $k$ and a Borel set $F \subset \mathbb{R}^{k}$. For a cylindrical set of the above kind let

$$
w(M)=(2 \pi)^{-k / 2} \prod_{i=1}^{k} \omega_{i}^{-1 / 2} \int_{F} e^{-(1 / 2) \sum_{i=1}^{k} \omega_{i}^{-1} z_{i}^{2}} d z_{1} \cdots d z_{k} .
$$

Then, it can be easily verified that the set of all cylindrical sets is an algebra and that the function $w$ is an additive measure on this algebra. In addition, $w(H)=1$. Further, it is known (see [10] for proofs) that the assumption that the operator $S$ is of trace class provides the countable additivity of the measure $w$ on the above algebra of all cylindrical sets. Therefore, this measure can be uniquely extended onto the minimal sigma-algebra $\mathcal{M}$ containing this algebra. In fact, $\mathcal{M}$ is the Borel sigma-algebra in the space $H$ (for proofs, see [10]). The measure $w$ considered on the sigma-algebra $M$ is called a centered Gaussian measure in the space $H$. It is essential for us that any centered Gaussian measure is a Radon measure, that is, for any Borel set $\Omega \subset H$ and for any $\epsilon>0$ there exists a compact set $K \subset \Omega$ such that $w(\Omega \backslash K)<\epsilon$. Finally, the following result is also useful for us (for the proof, see [27]).

STATEMENT 1. For any ball $B_{r}(a)=\left\{u \in H \mid\|u-a\|_{H}<r\right\}$, where $a \in H$ and $r>0$, one has $w\left(B_{r}(a)\right)>0$.

The problem (1.1), (1.2), and (1.3) can be rewritten in the equivalent real form for real functions $u$ and $v$, where $\psi=u+i v$, as follows:

$$
\begin{gathered}
u_{t}=-v_{x x}+2 \kappa\left(u^{2}+v^{2}\right) v, \quad x, t \in \mathbb{R}, \\
v_{t}=u_{x x}-2 \kappa\left(u^{2}+v^{2}\right) u, \quad x, t \in \mathbb{R}, \\
u(x+A, t)=u(x, t), \quad v(x+A, t)=v(x, t), \\
u\left(x, t_{0}\right)=u_{0}(x)=\operatorname{Re} \psi_{0}(x), \quad v\left(x, t_{0}\right)=v_{0}(x)=\operatorname{Im} \psi_{0}(x) .
\end{gathered}
$$

We suppose that $\psi=\psi(x, t)$ is a solution of the problem (1.1), (1.2), and (1.3) infinitely differentiable with respect to $x$ and $t$ and $(u(x, t), v(x, t))$ is the corresponding solution of the problem (2.5). Let $w_{1}=\psi(x, t)$ and

$$
w_{n+1}=-i \frac{d w_{n}}{d x}+\kappa \bar{\psi} \sum_{k=1}^{n-1} w_{k} w_{n-k}
$$

We set

$$
L_{n}(\psi)=L_{n}(u, v)=\int_{0}^{A} \bar{\psi}(x, t) w_{n}(x, t) d x,
$$

where $\psi=u+i v$ and $n=1,2,3, \ldots$. Then, the statement is that the quantities $L_{n}(\psi)=$ $L_{n}(u, v)$ (here $\psi=u+i v$ ) are independent of $t$ (see [22]) (i.e., the functionals $L_{n}$ are 
conservation laws for the problems (1.1), (1.2), (1.3), and (2.5)). Let

$$
Q_{n}(\psi)=Q_{n}(u, v)=\frac{1}{2} \operatorname{Re} L_{2 n+1}(\psi),
$$

where $n=0,1,2, \ldots$. Then, one can easily get from (2.6) and (2.7) that

$$
Q_{n}(\psi)=Q_{n}(u, v)=\int_{0}^{A}\left\{\frac{1}{2}\left|D_{x}^{n} \psi\right|^{2}+\frac{1}{2}|\psi|^{2}-q_{n}\left(\psi, \bar{\psi}, \ldots, D_{x}^{n-1} \psi, D_{x}^{n-1} \bar{\psi}\right)\right\} d x,
$$

where the functions $q_{n}$ are polynomials; in particular, they are infinitely differentiable. In what follows, we write $Q_{n}(\psi)=Q_{n}(u, v)$ and

$$
q_{n}\left(\psi, \bar{\psi}, \ldots, D_{x}^{n-1} \psi, D_{x}^{n-1} \bar{\psi}\right)=q_{n}\left(u, v, \ldots, D_{x}^{n-1} u, D_{x}^{n-1} v\right)
$$

for simplicity of the notation.

Methods of investigation of the well-posedness of the problems (1.1), (1.2), (1.3), and (2.5) are proposed in a large number of articles (cf. [12, 14, 23]). In our one-dimensional case $\left(x \in \mathbb{R}^{1}\right)$ these problems become essentially simpler. Now we formulate the result we need. Its proof is presented later.

THEOREM 2.1. For any positive integer $n$ and for any $\psi_{0} \in H^{n}$ and $T>0$ there exists a unique solution to the problem (1.1), (1.2), and (1.3) of the class $\psi(\cdot, t) \in$ $C\left(\left[t_{0}-T, t_{0}+T\right] ; H^{n}\right)$. For any fixed $t$ the function $\psi_{0} \rightarrow \psi(\cdot, t)$ is a homeomorphism as a map from $H^{n}$ into $H^{n}$. Further, $f_{1}^{n}\left(f_{1}^{n}(\psi, t), \tau\right)=f_{1}^{n}(\psi, t+\tau)$ for all $\psi \in H^{n}$ and $t, \tau \in \mathbb{R}$ where $f_{1}^{n}\left(\psi_{0}, t\right)=\psi\left(\cdot, t+t_{0}\right)$. In addition, the quantities $Q_{0}(\psi(\cdot, t)), \ldots$, $Q_{n}(\psi(\cdot, t))$ are independent of $t$ (i.e., they are conservation laws).

REMARK 2.2. Sometimes we call solutions given by Theorem 2.1 the $H^{n}$-solutions of the problem (1.1), (1.2), and (1.3) (or (3.1), see further).

Let $H_{\mathrm{Re}}^{n}$ be the subspace of the space $H^{n}$ consisting of real functions from $H^{n}$ and let $X_{n}=H_{\mathrm{Re}}^{n} \otimes H_{\mathrm{Re}}^{n}$ be the Cartesian product of two samples of this new space. We equip the space $X_{n}$ by the usual scalar product

$$
\left(\left(u_{1}, v_{1}\right),\left(u_{2}, v_{2}\right)\right)_{n}=\left(u_{1}, u_{2}\right)_{n}+\left(v_{1}, v_{2}\right)_{n} .
$$

Then, we introduce a selfadjoint operator $S_{n}$ of trace class acting in the space $X_{n}$ by the rule

$$
S_{n}=\left(\begin{array}{cc}
T^{-1} & 0 \\
0 & T^{-1}
\end{array}\right),
$$

where $T=\left(I+\Delta^{n+1}\right)\left(I+\Delta^{n}\right)^{-1}$. Take an arbitrary integer $n \geq 1$ and let $w^{n}$ be the centered Gaussian measure with the correlation operator $S_{n}$ in the space $X_{n}$. Now we can give the following new formulation of Theorem 2.1.

THEOREM 2.3. Let $\left(u_{0}, v_{0}\right) \in X_{n}$ where $n \geq 1$ is an integer number. Then, for any $T>0$ there exists a unique solution $(u(\cdot, t), v(\cdot, t)) \in C\left(\left[t_{0}-T, t_{0}+T\right] ; X_{n}\right)$ to the problem (2.5). For any fixed the function transforming $\left(u_{0}, v_{0}\right)$ into $(u(\cdot, t), v(\cdot, t))$ is a homeomorphism as a map from $X_{n}$ into $X_{n}$. If $f^{n}\left(u_{0}, v_{0}, t\right)=\left(u\left(t+t_{0}\right), v\left(t+t_{0}\right)\right)$ for all $\left(u_{0}, v_{0}\right) \in X_{n}$ where $(u(t), v(t))$ is the corresponding solution of the problem (2.5), 
then $f^{n}\left(f^{n}(u, v, \tau), t\right)=f^{n}(u, v, t+\tau)$ for all $(u, v) \in X_{n}$ and $t, \tau \in \mathbb{R}$. In addition, the quantities $Q_{0}(u(\cdot, t), v(\cdot, t)), \ldots, Q_{n}(u(\cdot, t), v(\cdot, t))$ are independent of $t$ for these solutions (i.e., they are conservation laws).

In what follows, we set $w(x, t)=(u(x, t), v(x, t))$ for an arbitrary solution $(u(x, t), v(x, t))$ of the problem (2.5). Not discussing various definitions of this concept, we accept that $f^{n}(w, t)$ is the DS with the phase space $X_{n}$. We only remark that if there exists a bounded IM $v$ for this DS so that $v(\Omega)=v\left(f^{n}(\Omega, t)\right)$ for any $t \in \mathbb{R}$ and a Borel set $\Omega \subset X_{n}$, then the Poincaré recurrence theorem takes place for the introduced DS (for details, see [19, 27]). (It should be remarked that, since the function $f^{n}(\cdot, t)$ is a homeomorphism as a map from $X_{n}$ into $X_{n}$, it transforms any Borel subset of the space $X_{n}$ into a Borel one.) For a Borel set $\Omega \subset X_{n}$ let

$$
\mu^{n}(\Omega)=\int_{\Omega} e^{\Phi_{n}(u, v)} d w^{n}(u, v),
$$

where $\Phi_{n}(u, v)=\int_{0}^{A} q_{n+1}\left(u, v, \ldots, D_{x}^{n} u, D_{x}^{n} v\right) d x$.

Our main result is the following.

THEOREM 2.4. For any positive integer $n, \mu^{n}$ is a Borel measure well defined in the space $X_{n}$ and it is an IM for the DS $f^{n}$. In addition,

$$
0<\mu^{n}\left(D_{R}\right)<\infty
$$

for all sufficiently large values $R>0$ where $D_{R}=\left\{w \in X_{n}|| Q_{0}(w)|<R, \ldots,| Q_{n}(w) \mid<\right.$ $R\}$. Thus, the sets $D_{R}$ can be taken for new phase spaces of the DS $f^{n}$.

We remark that we do not study the question whether the measure of the whole phase space is finite, that is, if it is right that $\mu^{n}\left(X_{n}\right)<\infty$, but we present in Theorem 2.4 a weaker statement sufficient for our aims. The following result is a corollary of Theorem 2.4, Statement 1 from this section and the Poincaré recurrence theorem (see [19, 27]).

THEOREM 2.5. For any positive integer $n$ consider the DS $f^{n}$. Then, a.a. points (with respect to the measure $w^{n}$ or $\mu^{n}$ ) of the phase space $X_{n}$ are stable according to Poisson. The set of points of the space $X_{n}$ stable according to Poisson is dense in $X_{n}$.

3. Proof of Theorem 2.1. We use standard methods of investigating the well-posedness of the Cauchy problem (1.1), (1.2), and (1.3) as in [12, 14, 23]. The idea of these methods is the replacement of the problem (1.1), (1.2), and (1.3) by the following abstract integral equation:

$$
\psi(t)=e^{-i\left(t-t_{0}\right) \Delta} \psi_{0}-2 i \kappa \int_{t_{0}}^{t} e^{-i(t-s) \Delta}\left[|\psi(s)|^{2} \psi(s)\right] d s .
$$

Here $\psi(t)$ is an abstract unknown function defined in segments $t \in I=\left[t_{0}-a, t_{0}+b\right]$, where $a, b>0$, with values in a functional space (to be interpreted as $H^{n}$ ). Formally, solutions of (3.1) satisfy the problem (1.1), (1.2), and (1.3). For a more complete information about relations between solutions of the problem (1.1), (1.2), and (1.3) and (3.1), see [14]. We accept the following definition. 
DEFINITION 3.1. We call solutions of (3.1) (generalized) solutions of the problem (1.1), (1.2), and (1.3).

We turn to proving Theorem 2.1. First, let $\psi_{0} \in H^{1}$, then the existence and uniqueness of a global (defined for all $t \in \mathbb{R}$ ) $H^{1}$-solution to (3.1) can be proved as in [12, 14] where the Cauchy problem (1.1), (1.2), and (1.3) is considered with initial data vanishing as $|x| \rightarrow \infty$; in addition, it can be proved as in these papers that $Q_{0}$ and $Q_{1}$ are conservation laws for $H^{1}$-solutions. The proof of the existence and uniqueness of a local $H^{n}$-solution to (3.1), where $n \geq 2$ is integer, repeats the procedure from these papers. So, we have that, for any integer $n \geq 2$ and $\psi_{0} \in H^{n}$, there exist $a>0$ and $b>0$ such that (3.1) has a unique solution $\psi(t) \in C\left(\left[t_{0}-a, t_{0}+b\right] ; H^{n}\right)$ and either this solution can be continued for all $t>t_{0}$ (resp., for all $t<t_{0}$ ) or there exists $b_{0}>0$ (resp., $a_{0}>0$ ) such that this solution can be continued onto the half-open interval $\left[t_{0}, t_{0}+b_{0}\right)$ (resp., onto the half-open interval $\left.\left(t_{0}-a_{0}, t_{0}\right]\right)$ and $\limsup _{t \rightarrow t_{0}+b_{0}-0}\|\psi(t)\|_{n}=\infty$ (resp., $\left.\limsup \operatorname{sut}_{t \rightarrow t_{0}-a_{0}+0}\|\psi(t)\|_{n}=\infty\right)$. In what follows, we show only that our $H^{n}$-solution $\psi(t)$ can be continued onto the whole half-line $\left[t_{0},+\infty\right)$ because the statement that it can be continued onto the half-line $\left(-\infty, t_{0}\right]$ can be proved by analogy.

Let $\psi_{0} \in H^{n}$ and $+\infty=T_{1} \geq T_{2} \geq T_{3} \geq \cdots \geq T_{n}>0$ be such numbers that $\left[t_{0}, t_{0}+T_{k}\right.$ ) are maximal half-open intervals of the existence of $H^{k}$-solutions of (3.1). For the above goal, it suffices to prove that $T_{1}=\cdots=T_{n}$. The following estimate easily follows from (3.1):

$$
\|\psi(t)\|_{k} \leq C_{1}\left\|\psi_{0}\right\|_{k}+C_{2}\left(\sup _{s \in\left[t_{0}, t\right]}\|\psi(s)\|_{l}\right) \int_{t_{0}}^{t}\|\psi(s)\|_{k} d s,
$$

where $l=k-1$ if $k \geq 2$ or $l=1$ if $k=1, C_{1}>0$ is independent of $t$ and $C_{2}(s)$ is a positive continuous function on the half-line $0 \leq s<+\infty$. The inequalities (3.2) easily imply the equality $T_{1}=\cdots=T_{n}$. Thus, the existence and uniqueness of a global $H^{n_{-}}$ solution $(n \geq 2)$ are proved.

Let $H=\cap_{n \geq 1} H^{n}=C^{\infty}$. Then, we have proved that for any $\psi_{0} \in H$ there exists a unique global $C^{\infty}$-solution to (3.1). It can be shown as in [14] that these $C^{\infty}$-solutions satisfy the problem (1.1), (1.2), and (1.3) and are infinitely differentiable with respect to $t$, too.

LEMmA 3.2. For any positive integer $n$ and $d>0$ there exists $R>0$ such that $\|g\|_{n}<$ $R$ for all $g \in H^{n}$ satisfying the conditions

$$
\left|Q_{0}(g)\right|<d, \ldots,\left|Q_{n}(g)\right|<d
$$

Proof. We use the known embedding theorem

$$
\|\mathfrak{g}\|_{L_{p}} \leq C\|g\|^{1 / 2+1 / p}\left(\left\|D_{x} \mathcal{g}\right\|+\|g\|\right)^{1 / 2-1 / p} \quad(p \geq 2)
$$

which takes place for all $g \in H^{1}$ with $C>0$ independent of $g$. Hence, using the functional $Q_{1}(g)+Q_{0}(g)=(1 / 2) \int_{0}^{A}\left\{\left|D_{x} g\right|^{2}+|g|^{2}+\kappa|g|^{4}\right\} d x$, we get the inequality

$$
\frac{1}{2}\|g\|_{1}^{2}-C\|g\|^{3}\|g\|_{1} \leq Q_{1}(g)+Q_{0}(g) \leq \frac{1}{2}\|g\|_{1}^{2}+C\|g\|^{3}\|g\|_{1}
$$


which implies that for any $d>0$ there exists $R>0$ such that $\|g\|_{1}<R$ for all $g \in H^{n}$ satisfying the condition $\left|Q_{1}(g)\right|+\left|Q_{0}(g)\right|<d$. Further, using (2.6), (2.7), and (2.9), we get

$$
\begin{aligned}
q_{n}(\psi, & \left.\bar{\psi}, \ldots, D_{x}^{n-1} \psi, D_{x}^{n-1} \bar{\psi}\right) \\
= & q_{n}^{11}\left(\psi, \bar{\psi}, \ldots, D_{x}^{n-2} \psi, D_{x}^{n-2} \bar{\psi}\right)\left(D_{x}^{n-1} u\right)^{2} \\
& +q_{n}^{12}\left(\psi, \bar{\psi}, \ldots, D_{x}^{n-2} \psi, D_{x}^{n-2} \bar{\psi}\right) D_{x}^{n-1} u D_{x}^{n-1} v \\
& +q_{n}^{22}\left(\psi, \bar{\psi}, \ldots, D_{x}^{n-2} \psi, D_{x}^{n-2} \bar{\psi}\right)\left(D_{x}^{n-1} v\right)^{2} \\
& +q_{n}^{1}\left(\psi, \bar{\psi}, \ldots, D_{x}^{n-2} \psi, D_{x}^{n-2} \bar{\psi}\right) D_{x}^{n-1} u \\
& +q_{n}^{2}\left(\psi, \bar{\psi}, \ldots, D_{x}^{n-2} \psi, D_{x}^{n-2} \bar{\psi}\right) D_{x}^{n-1} v \\
& +q_{n}^{0}\left(\psi, \bar{\psi}, \ldots, D_{x}^{n-2} \psi, D_{x}^{n-2} \bar{\psi}\right) .
\end{aligned}
$$

Hence, the inequalities

$$
Q_{k}(g) \geq \frac{1}{2}\|g\|_{k}^{2}-\gamma_{k}\left(\|g\|_{k-1}\right)
$$

take place for $k=\overline{2, n}$ (here $\gamma_{k}(s)$ are positive continuous functions). Now the statement of Lemma 3.2 follows from inequalities (3.5) and (3.7).

So, the functionals $Q_{0}, \ldots, Q_{n}$ are conservation laws for $H$-solutions of the problem (1.1), (1.2), and (1.3). Further, the inequality $(n \geq 1)$

$$
\begin{aligned}
\|\psi(t)-\varphi(t)\|_{n} \leq & C_{1}\left\|\psi_{0}-\varphi_{0}\right\|_{n}+C_{2}\left(\max _{s \in\left[t_{0}, t\right]}\|\psi(s)\|_{l} ; \max _{s \in\left[t_{0}, t\right]}\|\varphi(s)\|_{l}\right) \\
& \times \int_{t_{0}}^{t}\|\psi(s)-\varphi(s)\|_{n} d s
\end{aligned}
$$

(here $l=n-1$ if $n \geq 2$ or $l=1$ if $n=1$ ) following from (3.1) for arbitrary two $H^{n}$ solutions $\psi(t)$ and $\varphi(t)$ (where $\varphi\left(t_{0}\right)=\varphi_{0}$ ) of this equation, in view of Lemma 3.2 and the continuity of the functionals $Q_{0}, \ldots, Q_{n}$ in $H^{n}$ implies two corollaries:

(A) for $n \geq 1$ the functionals $Q_{0}, \ldots, Q_{n}$ are conservation laws for $H^{n}$-solutions of (3.1);

(B) $H^{n}$-solutions of (3.1) continuously depend on $\psi_{0}$, that is, for any $\psi_{0} \in H^{n}, \epsilon>0$ and $T>0$ there exists $\delta>0$ such that if $\left\|\psi_{0}-\psi_{0}^{\prime}\right\|_{n}<\delta$, then $\max _{t \in\left[t_{0}-T, t_{0}+T\right]}$ $\left\|\psi(t)-\psi^{\prime}(t)\right\|_{n}<\epsilon$ where $\psi^{\prime}(t)$ is the solution of (3.1) with $\psi_{0}=\psi_{0}^{\prime}$.

Finally, if $\psi(t)$ is a $H^{n}$-solution of (3.1) with $\psi_{0}=\psi\left(t_{0}\right)$ (here $n \geq 1$ ), then for any fixed $t \in \mathbb{R}$ the function $\psi(\tau)$ satisfies

$$
\psi(\tau)=e^{-i(\tau-t) \Delta} \psi(t)-2 i \kappa \int_{t}^{\tau} e^{-i(\tau-s) \Delta}\left[|\psi(s)|^{2} \psi(s)\right] d s
$$

Therefore, the function $\psi\left(t_{0}\right) \rightarrow \psi(t)$ is a one-to-one transformation of the space $H^{n}$ for any fixed $t$, hence it is a homeomorphism because it is continuous with the inverse. The property $f_{1}^{n}\left(f_{1}^{n}(\psi, t), \tau\right)=f_{1}^{n}(\psi, t+\tau)$ follows from these arguments, too. Theorem 2.1 is proved. 
4. An approximation of the NLS. In this section, we consider Galerkin approximations of the problem (1.1), (1.2), and (1.3). Here, among more or less standard results, we obtain certain statements crucial for us. These are Lemmas 4.4 and 4.5 and Proposition 4.7. In this section, all estimates with the use of the Gronwell lemma are made for $t>t_{0}$. Estimates for $t<t_{0}$ can be made by analogy.

Let

$$
\begin{gathered}
e_{0}(x)=\frac{1}{\sqrt{A}}, \quad e_{2 k-1}(x)=\sqrt{\frac{2}{A\left(1+\left(\lambda_{2 k-1}\right)^{n}\right)}} \sin \frac{2 \pi k x}{A}, \\
e_{2 k}=\sqrt{\frac{2}{A\left(1+\left(\lambda_{2 k}\right)^{n}\right)}} \cos \frac{2 \pi k x}{A},
\end{gathered}
$$

where $\lambda_{0}=0, \lambda_{2 k-1}=\lambda_{2 k}=(2 \pi k / A)^{2}(k=1,2,3, \ldots)$ are the eigenvalues of the operator $\Delta$. Then, $\left\{e_{k}\right\}_{k=0,1,2, \ldots}$ is the orthonormal basis in the space $H^{n}$ consisting of eigenfunctions of the operator $T$ with corresponding eigenvalues $\omega_{k}=\left(1+\lambda_{k}^{n+1}\right)\left(1+\lambda_{k}^{n}\right)^{-1}$ $(k=0,1,2, \ldots)$. Let $P_{m}$ be the orthogonal projector in the space $H^{n}$ onto the subspace $\operatorname{span}\left\{e_{0}, \ldots, e_{2 m}\right\}$. Let also $X^{m}=P_{m} H^{n}$.

Consider the following sequence of problems:

$$
\begin{gathered}
i \psi_{t}^{m}=-\psi_{x x}^{m}+2 \kappa P_{m}\left[\left|\psi^{m}\right|^{2} \psi^{m}\right], \quad x, t \in \mathbb{R}, \\
\psi_{0}^{m}=\psi^{m}\left(\cdot, t_{0}\right)=P_{m} \psi_{0} \quad(m=1,2,3, \ldots),
\end{gathered}
$$

or, equivalently, in terms of real functions $u^{m}$ and $v^{m}$ where $\psi^{m}=u^{m}+i v^{m}$ :

$$
\begin{aligned}
& u_{t}^{m}=-v_{x x}^{m}+2 \kappa P_{m}\left[\left(\left(u^{m}\right)^{2}+\left(v^{m}\right)^{2}\right) v^{m}\right], \\
& v_{t}^{m}=u_{x x}^{m}-2 \kappa P_{m}\left[\left(\left(u^{m}\right)^{2}+\left(v^{m}\right)^{2}\right) u^{m}\right], \\
& u_{0}^{m}=u^{m}\left(\cdot, t_{0}\right)=P_{m} u_{0}, \quad v_{0}^{m}=v^{m}\left(\cdot, t_{0}\right)=P_{m} v_{0} .
\end{aligned}
$$

First of all, we recall that in a finite-dimensional linear space any two norms are equivalent. Further, for any positive integer $m$ and $\psi_{0} \in H^{n}$ obviously there exist $a, b>0$ and a unique infinitely differentiable solution $\psi^{m}(x, t)$ to the problem (4.2) defined for $(x, t) \in \mathbb{R} \times(-a, b)$ and either $b>0$ is finite (resp., $a>0$ is finite) and $\limsup \operatorname{sut}_{t \rightarrow t_{0}+b-0}\left\|\psi^{m}(\cdot, t)\right\|_{n}=+\infty$ (resp., $a>0$ is finite and limsup $\operatorname{sut}_{t \rightarrow t_{0}-a+0}\left\|\psi^{m}(\cdot, t)\right\|_{n}$ $=+\infty$ ) or the solution $\psi^{m}$ can be continued onto the whole half-line $t>t_{0}$ (resp., onto the half-line $\left.t<t_{0}\right)$. Then, the direct verification shows that

$$
\frac{d}{d t} Q_{0}\left(\psi^{m}(\cdot, t)\right)=0
$$

for all $t$. This equality yields, in particular, that for any positive integer $m$ and $\psi_{0} \in H^{n}$ there exists $C>0$ such that

$$
\left\|\psi^{m}(\cdot, t)\right\|_{n} \leq C
$$

for all $t$ for which this solution is determined. Therefore, for any positive integer $m$ an arbitrary solution of the problem (4.2) can be continued onto the whole real line $t \in \mathbb{R}$. Consequently, any solution of the problem (4.3) and (4.4) can be continued onto the whole real line $t \in \mathbb{R}$. 
LEMMA 4.1. For an arbitrary segment $I=\left[t_{0}-T, t_{0}+T\right]$, where $T>0$, and for any positive integer $n$ there exists a function $\beta_{n}(s)$ continuous and nondecreasing on the half-line $[0,+\infty)$ such that for any $\psi_{0} \in H^{n}$ the inequality

$$
\left\|\psi^{m}(\cdot, t)\right\|_{n} \leq \beta_{n}\left(\left\|\psi_{0}^{m}\right\|_{n}\right)
$$

takes place for all $t \in I$ and for all $m=1,2,3, \ldots$ (here $\psi^{m}$ is the solution of the problem (4.2)).

Proof. First of all, one can easily verify that the functionals

$$
\begin{aligned}
& Q_{0}\left(\psi^{m}(\cdot, t)\right)=\frac{1}{2} \int_{0}^{A}\left|\psi^{m}(\cdot, t)\right|^{2} d x, \\
& Q_{1}\left(\psi^{m}(\cdot, t)\right)=\int_{0}^{A}\left\{\frac{1}{2}\left|D_{x} \psi^{m}(\cdot, t)\right|^{2}+\frac{\kappa}{2}\left|\psi^{m}(\cdot, t)\right|^{4}\right\} d x,
\end{aligned}
$$

are conservation laws for the problem (4.2), that is, they are independent of $t$. In addition, they are continuous functionals in $H^{n}$ bounded on bounded subsets of this space. Hence, as above, due to the known embedding theorem for functions $g \in H^{1}$

$$
\|g\|_{L_{p}} \leq C\|g\|^{1 / 2+1 / p}\left(\left\|g_{x}^{\prime}\right\|+\|g\|\right)^{1 / 2-1 / p},
$$

where $p \geq 2$, we have

$$
\begin{aligned}
& Q_{1}\left(\psi^{m}(\cdot, t)\right)+Q_{0}\left(\psi^{m}(\cdot, t)\right) \\
& \quad \geq \frac{1}{2}\left\|\psi^{m}(\cdot, t)\right\|_{1}^{2}-C\left\|\psi^{m}(\cdot, t)\right\|^{3}\left(\left\|D_{x} \psi^{m}(\cdot, t)\right\|+\left\|\psi^{m}(\cdot, t)\right\|\right) .
\end{aligned}
$$

Hence,

$$
\left\|\psi^{m}(\cdot, t)\right\|_{1} \leq \beta_{1}\left(\left\|\psi_{0}^{m}\right\|_{1}\right)
$$

for all $t \in \mathbb{R}$ where $\beta_{1}(s)$ is a function satisfying the properties indicated above for the function $\beta_{n}$.

Further, solutions of the problem (4.2) satisfy the following integral equation similar to (3.1):

$$
\psi^{m}(\cdot, t)=e^{-i\left(t-t_{0}\right) \Delta} P_{m} \psi_{0}-2 i \kappa \int_{t_{0}}^{t} e^{-i(t-s) \Delta} P_{m}\left[\left|\psi^{m}(\cdot, s)\right|^{2} \psi^{m}(\cdot, s)\right] d s .
$$

For $k=2, \ldots, n$ we get from $(4.12)$

$$
\left\|\psi^{m}(\cdot, t)\right\|_{k} \leq C_{1}\left\|\psi_{0}^{m}\right\|_{k}+C_{2}\left(\max _{t \in I}\left\|\psi^{m}(\cdot, t)\right\|_{k-1}\right) \int_{t_{0}}^{t}\left\|\psi^{m}(\cdot, s)\right\|_{k} d s .
$$

This inequality and (4.11) step by step lead to the statement of Lemma 4.1.

Proposition 4.2. Let $\psi_{0} \in H^{n}$, where $n$ is a positive integer, and let $T>0$ be an arbitrary number. Then,

$$
\lim _{m \rightarrow \infty} \max _{t \in\left[t_{0}-T, t_{0}+T\right]}\left\|\psi^{m}(\cdot, t)-\psi(\cdot, t)\right\|_{n}=0 \text { as } m \longrightarrow \infty,
$$

where $\psi(\cdot, t)$ is the solution of the problem (1.1), (1.2), and (1.3) given by Theorem 2.1 and $\psi^{m}$ is the solution of the problem (4.2). 
Proof. We get from (3.1), (4.12), and Lemmas 3.2 and 4.1

$$
\begin{aligned}
& \left\|\psi^{m}(\cdot, t)-\psi(\cdot, t)\right\|_{n} \\
& \quad \leq C_{1}\left\|\psi_{0}-P_{m} \psi_{0}\right\|_{n}+C_{2} \int_{t_{0}}^{t_{0}+T}\left\|P_{m}\left[|\psi(\cdot, s)|^{2} \psi(\cdot, s)\right]-|\psi(\cdot, s)|^{2} \psi(\cdot, s)\right\|_{n} d s \\
& \quad+C_{3}\left(\left\|\psi_{0}\right\|_{l}\right) \int_{t_{0}}^{t}\left\|\psi^{m}(\cdot, s)-\psi(\cdot, s)\right\|_{n} d s,
\end{aligned}
$$

where $l=n-1$ if $n>1$ and $l=1$ if $n=1$. The first term from the right-hand side of this inequality obviously tends to zero as $m \rightarrow \infty$. The integrand in the second term tends to zero for all $s$ and is uniformly bounded in $s$ and $m$ according to Lemma 3.2. Therefore, this second term tends to zero, too, as $m \rightarrow \infty$. Thus, the statement of Proposition 4.2 follows from this inequality.

COROLLARY 4.3. For any positive integer $n, \psi_{0} \in H^{n}$ and $t \in \mathbb{R}$

$$
\lim _{m \rightarrow \infty}\left[Q_{k}\left(\psi^{m}(\cdot, t)\right)-Q_{k}(\psi(\cdot, t))\right]=0 \quad \text { for } k=\overline{0, n}
$$

Proof. The proof follows from the continuity of functionals $Q_{k}, k=\overline{0, n}$, in the space $H^{n}$ and the proved Proposition 4.2.

As it is already noted, Lemmas 4.4 and 4.5 and Proposition 4.7 below are results of this section crucial for us. To establish them, we consider quantities $Q_{n+1}\left(\psi^{m}(\cdot, t)\right)$. In this connection, it should be remarked that, for $\psi_{0} \in H^{n}$, the expression $Q_{n+1}(\psi(\cdot, t))$ is not determined in general. However, since the functions $\psi^{m}$ are infinitely differentiable, the expression $Q_{n+1}\left(\psi^{m}(\cdot, t)\right)$ is well defined for each $m$. Moreover, Lemmas 4.4 and 4.5 and Proposition 4.7 take place.

LEMMA 4.4. Let $\psi_{0} \in H^{n}$ for a positive integer $n$ and $t \in \mathbb{R}$. Then,

$$
\lim _{m \rightarrow \infty}\left(\frac{d Q_{n+1}\left(\psi^{m}(\cdot, t)\right)}{d t}\right)=0
$$

Proof. First of all, obviously $P_{m} g \in C^{\infty}$ for any positive integer $m$ and $g \in H^{n}$. Then, since $Q_{n+1}$ is a conservation law for infinitely differentiable solutions of the problem (1.1), (1.2), and (1.3), substituting $i \psi_{x x}^{m}-2 i \kappa\left|\psi^{m}\right|^{2} \psi^{m}$ into the expression for $d Q_{n+1}\left(\psi^{m}(\cdot, t)\right) / d t$ in place of $\partial \psi^{m} / \partial t$, we get zero. Therefore, to get the right expression for $d Q_{n+1}\left(\psi^{m}(\cdot, t)\right) / d t$, we can bring the derivative with respect to $t$ into the integrand and substitute the expression $2 i \kappa P_{m}^{\perp}\left[\left|\psi^{m}(\cdot, t)\right|^{2} \psi^{m}(\cdot, t)\right]$ for $\partial \psi^{m}(\cdot, t) / \partial t$ (here $P_{m}^{\perp}=I-P_{m}$ where $I$ is the identity).

Using the representations (2.9) and (3.6) and the above arguments, we easily derive the following estimate:

$$
\left|\frac{d Q_{n+1}\left(\psi^{m}(\cdot, t)\right)}{d t}\right| \leq \gamma_{n+1}\left(\left\|\psi^{m}(\cdot, t)\right\| \|_{n}\right)|| P_{m}^{\perp}\left[\left|\psi^{m}(\cdot, t)\right|^{2} \psi^{m}(\cdot, t)\right] \|_{n},
$$


where $n \geq 1$ and $\gamma_{n+1}(s)$ is a continuous nondecreasing function of $s \in[0,+\infty)$. Since according to Proposition 4.2 and embedding theorems

$$
\begin{aligned}
\| P_{m}^{\perp} & {\left[\left|\psi^{m}(\cdot, t)\right|^{2} \psi^{m}(\cdot, t)\right] \|_{n} } \\
& \leq\left\|\left|\psi^{m}(\cdot, t)\right|^{2} \psi^{m}(\cdot, t)-|\psi(\cdot, t)|^{2} \psi(\cdot, t)\right\|_{n}+\left\|P_{m}^{\perp}\left[|\psi(\cdot, t)|^{2} \psi(\cdot, t)\right]\right\|_{n} \longrightarrow 0
\end{aligned}
$$

as $m \rightarrow \infty,(4.18)$ yields the statement of Lemma 4.4 .

LEMmA 4.5. For any positive integer $n$ and $t \in \mathbb{R}$ there exists a function $\eta_{n+1}(s)$, continuous and monotonically nondecreasing on the half-line $s \in[0,+\infty)$, such that

$$
\left|Q_{n+1}\left(\psi^{m}(\cdot, t)\right)-Q_{n+1}\left(\psi_{0}^{m}\right)\right| \leq \eta_{n+1}\left(\left\|\psi_{0}^{m}\right\|_{n}\right)
$$

for all positive integer $m$ and $\psi_{0} \in H^{n}$.

Proof. The proof follows from (4.18), Lemma 4.1, and the following inequality:

$$
\left|Q_{n+1}\left(\psi^{m}(\cdot, t)\right)-Q_{n+1}\left(\psi_{0}^{m}\right)\right| \leq \int_{t_{0}}^{t}\left|\frac{d Q_{n+1}\left(\psi^{m}(\cdot, s)\right)}{d s}\right| d s .
$$

Proposition 4.6. For any $\psi_{0} \in H^{n}$, where $n$ is a positive integer, any $\epsilon>0$ and $t \in \mathbb{R}$ there exists $\delta>0$ such that

$$
\left\|\psi^{m}(\cdot, t)-\psi_{1}^{m}(\cdot, t)\right\|_{n}<\epsilon
$$

for each $m=1,2,3, \ldots$ and for an arbitrary solution $\psi_{1}^{m}(\cdot, t)$ of problem (4.2) satisfying the condition

$$
\left\|\psi^{m}\left(\cdot, t_{0}\right)-\psi_{1}^{m}\left(\cdot, t_{0}\right)\right\|_{n}<\delta .
$$

Proof. We have from (4.12) and Lemma 4.1

$$
\begin{aligned}
& \left\|\psi^{m}(\cdot, t)-\psi_{1}^{m}(\cdot, t)\right\|_{n} \\
& \quad \leq C_{1} \delta+C_{2}\left(\left\|\psi^{m}\left(\cdot, t_{0}\right)\right\|_{l}\right) \times \int_{t_{0}}^{t}\left\|\psi^{m}(\cdot, s)-\psi_{1}^{m}(\cdot, s)\right\|_{n} d s,
\end{aligned}
$$

where $l=n-1$ for $n \geq 2$ and $l=1$ if $n=1$. This inequality step by step leads to the statement of Proposition 4.6.

The following statement together with Lemma 4.5 is used in the next section for proving Lemma 5.5 which is of principal importance for our proof of Theorem 2.4.

Proposition 4.7. Let $K \subset H^{n}$ be a compact set where $n$ is a positive integer. Then, for any $t \in \mathbb{R}$

$$
Q_{n+1}\left(\psi^{m}(\cdot, t)\right)-Q_{n+1}\left(\psi_{0}^{m}\right) \longrightarrow 0 \text { as } m \rightarrow \infty
$$

uniformly in $\psi_{0} \in K$ (here $\psi_{0}^{m}=P_{m} \psi_{0}$ and $\psi^{m}(\cdot, t)$ is the solution of the problem (4.2) with $\left.\psi^{m}\left(\cdot, t_{0}\right)=\psi_{0}^{m}\right)$. 
Proof. First of all, we prove that for any $\epsilon>0$ and $g \in K$ there exist $\delta>0$ and a number $m_{0}>0$ such that

$$
\left|Q_{n+1}\left(\psi^{m}(\cdot, t)\right)-Q_{n+1}\left(\psi_{0}^{m}\right)\right|<\epsilon
$$

for all $m \geq m_{0}$ and $\psi_{0} \in B_{\delta}(g)=\left\{h \in H^{n} \mid\|h-g\|_{n}<\delta\right\}$. From (4.18) and Lemma 4.1 we get

$$
\begin{aligned}
& \left|Q_{n+1}\left(\psi^{m}(\cdot, t)\right)-Q_{n+1}\left(\psi^{m}\left(\cdot, t_{0}\right)\right)\right| \\
& \quad \leq \int_{t_{0}}^{t} \gamma_{n+1}\left(\beta_{n}\left(\|g\|_{n}+\delta\right)\right) \times\left\|P_{m}^{\perp}\left[\left|\psi^{m}(\cdot, s)\right|^{2} \psi^{m}(\cdot, s)\right]\right\|_{n} d s .
\end{aligned}
$$

We estimate the integral from the right-hand side of (4.27). We have

$$
\begin{aligned}
\left\|P_{m}^{\perp}\left[\left|\psi^{m}(\cdot, s)\right|^{2} \psi^{m}(\cdot, s)\right]\right\|_{n} \leq & \left\|\left|\psi^{m}(\cdot, s)\right|^{2} \psi^{m}(\cdot, s)-\left|\bar{\psi}^{m}(\cdot, s)\right|^{2} \bar{\psi}^{m}(\cdot, s)\right\|_{n} \\
& +\left\|\left|\bar{\psi}^{m}(\cdot, s)\right|^{2} \bar{\psi}^{m}(\cdot, s)-|\bar{\psi}(\cdot, s)|^{2} \bar{\psi}(\cdot, s)\right\|_{n} \\
& +|| P_{m}^{\perp}\left[|\bar{\psi}(\cdot, s)|^{2} \bar{\psi}(\cdot, s)\right] \|_{n},
\end{aligned}
$$

where $\bar{\psi}^{m}$ and $\bar{\psi}$ are the solutions of the problems (4.2) and (1.1), (1.2), and (1.3), respectively, with $\psi_{0}=g$. The second and third terms in the right-hand side of this inequality are independent of $\psi_{0} \in B_{\delta}(g)$ and tend to zero as $m \rightarrow \infty$. Further, according to Proposition 4.6, for any $\epsilon>0$ and $t \in \mathbb{R}$ there exists $\delta>0$ such that the first term in the right-hand side of this inequality is smaller than $\epsilon / 2$ if $\psi_{0} \in B_{\delta}(g)$, for all $m$. Hence, we have proved that the expression under the integral in the right-hand side of the inequality (4.27) for any fixed $s$ becomes arbitrary small for sufficiently small $\delta>0$ and sufficiently large numbers $m$ if $\psi_{0} \in B_{\delta}(g)$.

Further, take into account Lemma 4.1 according to which the expression

$$
\left\|P_{m}^{\perp}\left[\left|\psi^{m}(\cdot, s)\right|^{2} \psi^{m}(\cdot, s)\right]\right\|_{n}
$$

is bounded uniformly with respect to $\psi_{0} \in K, m=1,2,3, \ldots$, and $s \in\left[t_{0}, t\right]$. Therefore, for any $g \in K$ and $\epsilon>0$ there exist $\delta>0$ and a number $m_{0}$ such that the right-hand side of (4.27) is smaller than $\epsilon$ if $\psi_{0} \in B_{\delta}(g)$ and $m \geq m_{0}$. So, we fix an arbitrary $\epsilon>0$ and for any $g \in K$ take $\delta=\delta(g)>0$ and $m_{0}=m_{0}(g)>0$ such that

$$
\left|Q_{n+1}\left(\psi^{m}(\cdot, t)\right)-Q_{n+1}\left(\psi_{0}^{m}\right)\right|<\epsilon
$$

if $\psi_{0} \in B_{\delta(g)}(g)$ and $m \geq m_{0}(g)$ ( $\delta$ and $m_{0}$ exist according to the above arguments). Then, since $K$ is a compact set, there exists its finite covering by balls $B_{\delta\left(g_{1}\right)}\left(g_{1}\right), \ldots$, $B_{\delta\left(g_{l}\right)}\left(g_{l}\right)$. Let $m_{1}=\max \left\{m_{0}\left(g_{1}\right), \ldots, m_{0}\left(g_{l}\right)\right\}$. Then, obviously, (4.30) is valid for all $\psi_{0} \in K$ if $m \geq m_{1}$. Thus, Proposition 4.7 is proved.

5. Proof of Theorem 2.4. There is an analogy between the proof of the invariance of the measures $\mu^{n}$ presented below and proofs of the invariance of measures associated with the energy conservation law in [25, 26, 27]. One of the principal differences 
between our construction here and the approaches in the mentioned papers consists, in particular, in the fact that, unlike in [25, 26, 27], in the present paper, generally speaking, the finite-dimensional measures $\mu_{m}$ (see below) are not invariant for the corresponding finite-dimensional dynamical systems. All the information from the general measure theory to be used further is contained in [13], for example. Then, we remark that all the results from Section 4 are valid for problem (4.3) and (4.4) with the corresponding reformulation. We fix an arbitrary positive integer $n$. Let $g_{1}=\left(e_{0}, 0\right), g_{2}=\left(0, e_{0}\right), g_{3}=\left(e_{1}, 0\right), g_{4}=\left(0, e_{1}\right), \ldots, g_{2 k+1}=\left(e_{k}, 0\right), g_{2 k+2}=\left(0, e_{k}\right), \ldots$ Then, $\left\{g_{k}\right\}_{k=1,2,3, \ldots}$ is the orthonormal basis consisting of the eigenfunctions of the operator $S_{n}$ in the space $X_{n}$. Let $L_{m}=\operatorname{span}\left\{g_{1}, \ldots, g_{4 m+2}\right\}$ be the sequence of finitedimensional subspaces of the space $X_{n}(m=1,2,3, \ldots)$. In the spaces $L_{m}$ we introduce the following finite-dimensional Gaussian measures:

$$
w_{m}(\Omega)=(2 \pi)^{-(2 m+1)} \prod_{k=0}^{2 m} \omega_{k} \int_{F} e^{-(1 / 2) \sum_{k=0}^{2 m} \omega_{k}\left(p_{k}^{2}+q_{k}^{2}\right)} d p_{0} d q_{0} \cdots d p_{2 m} d q_{2 m},
$$

where $\Omega=\left\{u \in L_{m} \mid\left[\left(u, g_{1}\right)_{n}, \ldots,\left(u, g_{4 m+2}\right)_{n}\right] \in F\right\}$ and $F \subset \mathbb{R}^{4 m+2}$ is a Borel set. So, $w_{m}$ is a centered Gaussian measure in the space $L_{m}$ for any positive integer $m$. We recall that the space $L_{m}$ is equipped with the topology generated by the norm of the space $X_{n}$.

Further, let $w_{m}(\Omega)=w_{m}\left(\Omega \cap L_{m}\right)$ for any Borel subset $\Omega$ of the space $X_{n}$. We state that, with this definition, the measure $w_{m}$ becomes a well-defined Borel measure in the space $X_{n}$. To prove this statement, it suffices to show that $\Omega \cap L_{m}$ is a Borel subset of the space $L_{m}$ for any Borel set $\Omega \subset X_{n}$. Indeed, it is clear that the set $\mathscr{K}$ of all subsets of the space $L_{m}$ of this kind is a sigma-algebra of subsets of the space $L_{m}$ which obviously contains all open subsets of this space. Therefore, it suffices to prove that $\mathscr{K}$ is the minimal sigma-algebra of subsets of the space $L_{m}$ containing all open subsets of this space. Suppose that this is not right. Then, the Borel sigma-algebra $\mathscr{K}_{1}$ of the space $L_{m}$ is contained in the sigma-algebra $\mathscr{K}_{\mathrm{K}}$ and $\mathscr{K}_{1} \neq \mathscr{K}$. Consider the set $\mathcal{N}$ of all Borel subsets $A$ of the space $X_{n}$ such that $A \cap L_{m} \in \mathscr{K}_{1}$. Then, it is clear that $\mathcal{N}$ is a sigma-algebra in the space $X_{n}$ which contains all open subsets of the space $X_{n}$ and is more narrow than the Borel sigma-algebra of this space. Thus, we arrive at a contradiction and, therefore, we have proved that $\Omega \cap L_{m}$ is a Borel subset of the space $L_{m}$ for an arbitrary Borel subset $\Omega$ of the space $X_{n}$.

LEMмA 5.1. The sequence $\left\{w_{m}\right\}_{m=1,2,3, \ldots}$ of the Borel measures weakly converges to the measure $w^{n}$ in the space $X_{n}$.

REMARK 5.2. We recall that, by definition, a sequence of bounded Borel measures $\left\{v_{k}\right\}_{k=1,2,3, \ldots}$ weakly converges to a bounded Borel measure $v$ in a separable complete metric space $H$ if

$$
\lim _{k \rightarrow \infty} \int_{H} \Phi(g) d v_{k}(g)=\int_{H} \Phi(g) d v(g)
$$

for an arbitrary real continuous bounded functional $\Phi$ in the space $H$.

REMARK 5.3. A statement similar to Lemma 5.1 is presented in [27]; here we make some additions to its proof from that paper. 
Proof of Lemma 5.1. As it is proved in [27], the sequence of measures $\left\{w_{m}\right\}$ is weakly compact in the space $X_{n}$. In addition, for any cylindrical set $M \subset X_{n}$ we have $w_{m}(M)=w^{n}(M)$ for sufficiently large numbers $m$.

Suppose that the sequence $\left\{w_{m}\right\}$ does not converge weakly to the measure $w^{n}$. Then, there exists a Borel measure $w^{\prime}$ in $X_{n}$, not coinciding with the measure $w^{n}$, and a subsequence $\left\{w_{m_{k}}\right\}$ of the sequence $\left\{w_{m}\right\}$ such that the sequence $\left\{w_{m_{k}}\right\}$ weakly converges to the measure $w^{\prime}$. Further, since the extension of a measure, countably additive on an algebra, to the minimal sigma-algebra containing this algebra is unique (see [13]), there exists a cylindrical set of the kind

$$
M=\left\{g \in X_{n} \mid\left[\left(g, g_{1}\right)_{n}, \ldots,\left(g, g_{4 r+2}\right)_{n}\right] \in F\right\},
$$

where $r$ is a positive integer and $F \subset \mathbb{R}^{4 r+2}$ is a Borel set, such that $w^{\prime}(M) \neq w^{n}(M)$. Then, due to the known property of Borel sets, there exists a sequence $F_{1} \supset F_{2} \supset \cdots \supset$ $F_{i} \supset \cdots$ of open sets $F_{i} \subset \mathbb{R}^{4 r+2}$ such that $F=\bigcap_{i \geq 1} F_{i}$. Therefore,

$$
w^{\prime}(M)=\lim _{i \rightarrow \infty} w^{\prime}\left(M_{i}\right), \quad w^{n}(M)=\lim _{i \rightarrow \infty} w^{n}\left(M_{i}\right),
$$

where

$$
M_{i}=\left\{g \in X_{n} \mid\left[\left(g, g_{1}\right)_{n}, \ldots,\left(g, g_{4 r+2}\right)_{n}\right] \in F_{i}\right\} .
$$

Hence, we can accept that the cylindrical set $M \subset X_{n}$ is open.

Take an arbitrary $\epsilon>0$ and consider a continuous functional $\Phi_{\epsilon}(z)=\Phi_{\epsilon}\left(z_{1} g_{1}+\right.$ $\left.\cdots+z_{4 r+2} g_{4 r+2}\right)$ depending on variables $z=\left(z_{1}, \ldots, z_{4 r+2}\right) \in \mathbb{R}^{4 r+2}$ and satisfying the following properties:

(1) $0 \leq \Phi_{\epsilon}(z) \leq 1$ for all $z$;

(2) $\Phi_{\epsilon}(z)=0$ if $g=z_{1} g_{1}+\cdots+z_{4 r+2} g_{4 r+2} \notin M$;

(3) $\Phi_{\epsilon}(z)=1$ if $g \in M$ and $\operatorname{dist}(g ; \partial M) \geq \epsilon$ where for an arbitrary set $A \subset X_{n}$ and $g \in X_{n} \operatorname{dist}(g ; A)=\inf _{h \in A}\|g-h\|_{n}$.

It is clear that

$$
\int_{X_{n}} \Phi_{\epsilon}(z) d w_{m}(g)=\int_{X_{n}} \Phi_{\epsilon}(z) d w^{n}(g)
$$

for $m \geq r$. Further, $\lim _{\epsilon \rightarrow+0} \int_{X_{n}} \Phi_{\epsilon}(z) d w^{n}(g)=w^{n}(M)$. At the same time, there exists $\epsilon>0$ such that each of the expressions

$$
\begin{gathered}
\left|w^{n}(M)-\int_{X_{n}} \Phi_{\epsilon}(z) d w^{n}(g)\right| \\
\left|w^{\prime}(M)-\lim _{k \rightarrow \infty} \int_{X_{n}} \Phi_{\epsilon}(z) d w_{m_{k}}(g)\right|=\left|w^{\prime}(M)-\int_{X_{n}} \Phi_{\epsilon}(z) d w^{\prime}(g)\right|
\end{gathered}
$$

is smaller than $\left|w^{\prime}(M)-w^{n}(M)\right| / 3$. This fact contradicts (5.6). Thus, Lemma 5.1 is proved.

We introduce the following measures:

$$
\mu_{m}(\Omega)=\int_{\Omega} e^{\Phi_{n}(u, v)} d w_{m}(u, v)
$$


(here $\Omega \subset X_{n}$ are Borel sets). Since due to the representations (2.9) and (3.6) the functional $\Phi_{n}(u, v)$ is continuous in $X_{n}$ and bounded on any bounded subset of the space $X_{n}$, the measures $\mu_{m}$ and $\mu^{n}$ are well defined in $X_{n}$.

With the following statement, we in fact establish a well-known property of weakly converging sequences of measures.

LEMMA 5.4. One has $\liminf _{m \rightarrow \infty} \mu_{m}(\Omega) \geq \mu^{n}(\Omega)$ for any open bounded set $\Omega \subset X_{n}$ and $\limsup _{m \rightarrow \infty} \mu_{m}(K) \leq \mu^{n}(K)$ for any closed bounded set $K \subset X_{n}$.

For the proof, see, for example, [27].

Let $f_{m}\left(u_{0}, v_{0}, t\right)=\left(u^{m}\left(\cdot, t+t_{0}\right), v^{m}\left(\cdot, t+t_{0}\right)\right)$ for any $\left(u_{0}, v_{0}\right) \in X_{n}$ and $t \in \mathbb{R}$ where $\left(u^{m}(\cdot, t), v^{m}(\cdot, t)\right)$ is the solution of problem (4.3) and (4.4). One of the principal differences between the constructions in the present paper and in earlier papers [25, 26, 27] is that, unlike in $[25,26,27]$, in the present case the finite-dimensional measures $\mu_{m}$ are not in general invariant for the corresponding DS $f_{m}$. In this connection, we note that the following statement is crucial for us; it shows that the measures $\mu_{m}$ are "almost invariant" asymptotically for sufficiently large numbers $m$.

LEMmA 5.5. Let $t \in \mathbb{R}$ and $\Omega \subset X_{n}$ be an arbitrary closed bounded set. Then,

$$
\lim _{m \rightarrow \infty}\left[\mu_{m}\left(f_{m}(\Omega, t)\right)-\mu_{m}(\Omega)\right]=0 .
$$

Proof. We rewrite system (4.3) for coordinates $p(t)=\left(p_{0}(t), \ldots, p_{2 m}(t)\right)$ and $q(t)=$ $\left(q_{0}(t), \ldots, q_{2 m}(t)\right)$ where $u^{m}(x, t)=\sum_{k=0}^{2 m} p_{k}(t) e_{k}(x)$ and $v^{m}(x, t)=\sum_{k=0}^{2 m} q_{k}(t) e_{k}(x)$. Then, we get the following system of ordinary differential equations

$$
\begin{aligned}
& \dot{p}_{k}(t)=\frac{\partial h(p, q)}{\partial q_{k}}\left(1+\lambda_{k}^{n}\right), \quad k=\overline{0,2 m}, \\
& \dot{q}_{k}(t)=-\frac{\partial h(p, q)}{\partial p_{k}}\left(1+\lambda_{k}^{n}\right), \quad k=\overline{0,2 m} .
\end{aligned}
$$

Here $h(p, q)=Q_{1}\left(u^{m}, v^{m}\right)=(1 / 2) \int_{0}^{A}\left\{\left(u_{x}^{m}\right)^{2}+\left(v_{x}^{m}\right)^{2}+\kappa\left(\left(u^{m}\right)^{2}+\left(v^{m}\right)^{2}\right)^{2}\right\} d x$. Therefore, according to the well-known result [19, 27], the Lebesgue measure $\sigma_{m}$ in the phase space $\mathbb{R}^{4 m+2}$ is an IM for this DS (in fact, this is a well-known statement according to which the Lebesgue measure defined in the phase space of a finite-dimensional Hamiltonian system is an IM for this system). This easily implies that

$$
D=\left|\operatorname{det}\left\{\frac{\partial(p(t), q(t))}{\partial(p(0), q(0))}\right\}\right| \equiv 1
$$

for an arbitrary solution $(p(t), q(t))$ of (5.10).

Fix an arbitrary bounded closed set $\Omega \subset X_{n}$. Then, according to the above arguments, we have

$$
\mu_{m}\left(f_{m}(\Omega, t)\right)=\int_{\Omega} e^{Q_{n+1}\left(P_{m} w\right)-Q_{n+1}\left(f_{m}(w, t)\right)} d \mu_{m}(w) .
$$

Further,

$$
\left|\mu_{m}(\Omega)-\mu_{m}\left(f_{m}(\Omega, t)\right)\right| \leq \int_{\Omega}\left|1-e^{Q_{n+1}\left(P_{m} w\right)-Q_{n+1}\left(f_{m}(w, t)\right)}\right| d \mu_{m}(w) .
$$


According to Lemma 4.5, the integrand here is uniformly bounded with respect to $w \in \Omega$ by a constant $C>0$. Fix an arbitrary $\epsilon>0$. Then, according to the Prokhorov's theorem (see [10]), there exists a compact set $K \subset \Omega$ such that $\mu_{m}(\Omega \backslash K)<\epsilon$ for all $m=1,2,3, \ldots$ and $\mu^{n}(\Omega \backslash K)<\epsilon$. Further, in view of Proposition 4.7 and (5.13),

$$
\lim _{m \rightarrow \infty}\left[\mu_{m}(K)-\mu_{m}\left(f_{m}(K, t)\right)\right]=0
$$

therefore

$$
\limsup _{m \rightarrow \infty}\left|\mu_{m}(\Omega)-\mu_{m}\left(f_{m}(\Omega, t)\right)\right| \leq C \epsilon,
$$

where $C>0$ depends only on $\sup _{g \in \Omega}\|g\|_{n}$. Hence, due to the arbitrariness of $\epsilon>0$, Lemma 5.5 is proved.

COROLLARY 5.6. For any bounded open set $\Omega \subset X_{n}$ and $t \in \mathbb{R}$,

$$
\lim _{m \rightarrow \infty}\left[\mu_{m}(\Omega)-\mu_{m}\left(f_{m}(\Omega, t)\right)\right]=0
$$

The further proof of Theorem 2.4 including Lemma 5.7 below in fact repeats a construction in $[25,26,27]$.

LEMmA 5.7. Let $\Omega \subset X_{n}$ be an open bounded set and $t \in \mathbb{R}$. Then, $\mu^{n}(\Omega)=$ $\mu^{n}\left(f^{n}(\Omega, t)\right)$.

Proof. According to Theorem 2.3 and Lemma 3.2, $f^{n}(\Omega, t)$ is a bounded open subset of the space $X_{n}$. Fix an arbitrary $\epsilon>0$. Then, since $\mu^{n}$ is a Radon measure, there exists a compact set $K \subset \Omega$ such that $\mu^{n}(\Omega \backslash K)<\epsilon$. Let $K_{1}=f^{n}(K, t)$. Then, $K_{1}$ is a compact set, too, and $K_{1} \subset f^{n}(\Omega, t)=\Omega_{1}$. Let $\alpha=\min \left\{\operatorname{dist}(K, \partial \Omega) ; \operatorname{dist}\left(K_{1}, \partial \Omega_{1}\right)\right\}$ where $\operatorname{dist}(A, B)=\inf _{u \in A ; v \in B}\|u-v\|_{n}$ for any $A, B \subset X_{n}$ and $\partial A$ is the boundary of the set $A$. Obviously $\alpha>0$. Due to Proposition 4.6 for any $g \in K$ there exists a ball $B_{r}(g)=\left\{h \in X_{n} \mid\|g-h\|_{n}<r\right\} \subset \Omega$ with a positive radius $r$ such that

$$
\left\|f_{m}(g, t)-f_{m}(h, t)\right\|_{n}<\frac{\alpha}{3}
$$

for all $h \in B_{r}(g)$ and for all $m$. Let $B_{1}, \ldots, B_{l}$ be a finite covering of the compact set $K$ by these balls. Let $\Omega_{1}^{\beta}=\left\{h \in \Omega_{1} \mid \operatorname{dist}\left(h, \partial \Omega_{1}\right) \geq \beta\right\}$ where $\beta>0$, and $D=\bigcup_{i=1}^{l} B_{i}$. Since in view of Proposition $4.2 f_{m}(g, t) \rightarrow f^{n}(g, t)$ in $X_{n}$ as $m \rightarrow \infty$ for any $g \in X_{n}$, we get

$$
f_{m}(D, t) \subset \Omega_{1}^{\alpha / 4}
$$

for all sufficiently large numbers $m$.

Further, according to Lemmas 5.4, 5.5, and Corollary 5.6

$$
\begin{aligned}
\mu^{n}(\Omega) & \leq \mu^{n}(D)+\epsilon \leq \liminf _{m \rightarrow \infty} \mu_{m}(D)+\epsilon \\
& =\liminf _{m \rightarrow \infty} \mu_{m}\left(f_{m}(D, t)\right)+\epsilon \\
& \leq \mu^{n}\left(\Omega_{1}^{\alpha / 4}\right)+\epsilon \leq \mu^{n}\left(\Omega_{1}\right)+\epsilon
\end{aligned}
$$


Therefore, due to the arbitrariness of $\epsilon>0$, we have $\mu^{n}(\Omega) \leq \mu^{n}\left(\Omega_{1}\right)$. By analogy, $\mu^{n}(\Omega) \geq \mu^{n}\left(\Omega_{1}\right)$. Hence, $\mu^{n}(\Omega)=\mu^{n}\left(\Omega_{1}\right)$ and Lemma 5.7 is proved.

We prove Theorem 2.4. Let first $\Omega \subset X_{n}$ be an open (unbounded) set. Let $\Omega^{k}=\{w \in$ $\left.\Omega \mid\left\|f^{n}(w, t)\right\|_{n}+\|w\|_{n}<k\right\}$. Then, $\Omega=\bigcup_{k=1}^{\infty} \Omega^{k}$ and each set $\Omega^{k}$ is bounded and open. In addition, $f^{n}(\Omega, t)=\bigcup_{k=1}^{\infty} f^{n}\left(\Omega^{k}, t\right)$ and $\mu^{n}\left(\Omega^{k}\right)=\mu^{n}\left(f^{n}\left(\Omega^{k}, t\right)\right)$. Therefore,

$$
\mu^{n}\left(f^{n}(\Omega, t)\right)=\lim _{k \rightarrow \infty} \mu^{n}\left(f^{n}\left(\Omega^{k}, t\right)\right)=\lim _{k \rightarrow \infty} \mu^{n}\left(\Omega^{k}\right)=\mu^{n}(\Omega) .
$$

Further, for an arbitrary Borel subset $A$ of the space $X_{n}$ we get the equality $\mu^{n}(A)=$ $\mu^{n}\left(f^{n}(A, t)\right)$ approximating this set $A$ from outside by open sets (we remark that, since the function $f^{n}(\cdot, t)$ is a homeomorphism for any fixed $t$, it transforms Borel sets into Borel ones). The last statement of Theorem 2.4 follows from Lemma 3.2. Theorem 2.4 is proved.

REMARK 5.8. As it was noted in the introduction, an IM for the NLS generated by the conservation law $Q_{1}$ is constructed in [5], for example. Here, we presented IMs generated by the conservation laws $Q_{n}$ where $n \geq 2$. The question is left open whether there is an IM associated with the conservation law $Q_{0}$. As one could observe from our considerations, to any conservation law $Q_{n}(n \geq 2)$ there corresponds an IM on the phase space $H^{n-1}$. Therefore, it seems to be probable that such an IM could exist on a phase space like $H^{-1}$. However, since the well-posedness of the problem (1.1), (1.2), and (1.3) in a similar sense seems to be open and very difficult, we could not make a construction like that.

ACKNowledgement. The author is thankful to Dr A. P. Isaev for useful discussions and to Mrs T. Dumbrajs for editing the text.

\section{REFERENCES}

[1] A. A. Arsen'ev, Invariant measures for classical dynamical systems with infinite phase space, Mat. Sb. (N.S.) 121(163) (1983), no. 3, 297-309 (Russian), [translated in Math. USSR-Sb. 49 (1984), 291-303. Zbl 0542.58007]. MR 84j:82006.

[2] B. Bidégaray, Invariant measures for some partial differential equations, Phys. D 82 (1995), no. 4, 340-364. MR 96a:58172. Zbl 0888.58021.

[3] J. Bourgain, Fourier transform restriction phenomena for certain lattice subsets and applications to nonlinear evolution equations. I. Schrödinger equations, Geom. Funct. Anal. 3 (1993), no. 2, 107-156. MR 95d:35160a. Zbl 0787.35097.

[4] , Fourier transform restriction phenomena for certain lattice subsets and applications to nonlinear evolution equations. II. The KdV-equation, Geom. Funct. Anal. 3 (1993), no. 3, 209-262. MR 95d:35160b. Zbl 0787.35098.

[5]__ Periodic nonlinear Schrödinger equation and invariant measures, Comm. Math. Phys. 166 (1994), no. 1, 1-26. MR 95k:35185. Zbl 0822.35126.

[6] G. Casati, I. Guarneri, and F. Valz-Gris, Preliminaries to the ergodic theory of infinitedimensional systems: a model of radiant cavity, J. Statist. Phys. 30 (1983), no. 1, 195-217. MR 84k:82011.

[7] I. D. Chueshov, Equilibrium statistical solutions for dynamical systems with an infinite number of degrees of freedom, Math. USSR-Sb. 58 (1987), 397-406, [translated from Mat. Sb. (N.S.) 130(172) (1986), no. 3, 394-403 (Russian). MR 88b:81097]. Zbl 0632.60108 . 
, On the structure of equilibrium states of a class of dynamical systems connected with Lie-Poisson brackets, Teoret. Mat. Fiz. 75 (1988), no. 3, 445-450 (Russian). MR 90g:82007. Zbl 0659.58011.

[9] V. A. Chulaevskiū, The inverse problem method of scattering theory in statistical physics, Funktsional. Anal. i Prilozhen. 17 (1983), no. 1, 53-62 (Russian). MR 84j:82007.

[10] Y. L. Daletskiĭ and S. V. Fomin, Mery i differentsialnye uravneniya $v$ beskonechnomernykh prostranstvakh [Measures and Differential Equations in Infinite-Dimensional Spaces], Nauka, Moscow, 1983 (Russian). MR 86g:46059. Zbl 0536.46031.

[11] L. Friedlander, An invariant measure for the equation $u_{t t}-u_{x x}+u^{3}=0$, Comm. Math. Phys. 98 (1985), no. 1, 1-16. MR 87h:35293. Zbl 0576.35082.

[12] J. Ginibre and G. Velo, On a class of nonlinear Schrödinger equations. I. The Cauchy problem, general case, J. Funct. Anal. 32 (1979), no. 1, 1-32. MR 82c:35057. Zbl 0396.35028.

[13] P. H. Halmos, Measure Theory, Graduate Texts in Mathematics, vol. 18, Springer, New York, 1974. Zbl 0283.28001.

[14] T. Kato, On nonlinear Schrödinger equations, Ann. Inst. H. Poincaré Phys. Théor. 46 (1987), no. 1, 113-129. MR 88f:35133. Zbl 0632.35038.

[15] J. L. Lebowitz, H. A. Rose, and E. R. Speer, Statistical mechanics of the nonlinear Schrödinger equation, J. Statist. Phys. 50 (1988), no. 3-4, 657-687. MR 89f:82006. Zbl 0925.35142.

[16] H. P. McKean, Statistical mechanics of nonlinear wave equations. IV. Cubic Schrödinger, Comm. Math. Phys. 168 (1995), no. 3, 479-491. MR 96k:35182a. Zbl 0821.60069.

[17] H. P. McKean and E. Trubowitz, Hill's operator and hyperelliptic function theory in the presence of infinitely many branch points, Comm. Pure Appl. Math. 29 (1976), no. 2, 143-226. MR 55\#761. Zbl 0339.34024.

[18] H. P. McKean and K. L. Vaninsky, Statistical mechanics of nonlinear wave equations, Trends and Perspectives in Applied Mathematics (L. Sirovich, ed.), Appl. Math. Sci., vol. 100, Springer, New York, 1994, pp. 239-264. MR 95h:35234. Zbl 0811.35171.

[19] V. Nemytskii and V. Stepanov, Qualitative Theory of Differential Equations, Gostekhizdat, Moscow, Leningrad, 1949 (Russian).

[20] R. S. Palais, The symmetries of solitons, Bull. Amer. Math. Soc. (N.S.) 34 (1997), no. 4, 339-403. MR 98f:58111. Zbl 0886.58040.

[21] A. V. Skorokhod, Integration in Hilbert Space, Nauka, Moscow, 1975 (Russian).

[22] L. A. Takhtadzhyan and L. D. Faddeev, Gamiltonov podkhod v teorii solitonov [The Hamiltonian Approach in Soliton Theory], Nauka, Moscow, 1986 (Russian). MR 89m:58102. Zbl 0632.58003.

[23] Y. Tsutsumi, $L^{2}$-solutions for nonlinear Schrödinger equations and nonlinear groups, Funkcial. Ekvac. 30 (1987), no. 1, 115-125. MR 89c:35143. Zbl 0638.35021.

[24] P. E. Zhidkov, An invariant measure for the nonlinear Schrödinger equation, Dokl. Akad. Nauk SSSR 317 (1991), no. 3, 543-546 (Russian), [translated in Soviet Math. Dokl. 43 (1991), no. 2, 431-434. Zbl 0806.35168]. MR 92g:35203.

[25] _ _ An invariant measure for a nonlinear Schrödinger equation, JINR Commun. (1994), P5-94-199 (Russian).

[26] _ An invariant measure for a nonlinear wave equation, Nonlinear Anal. 22 (1994), no. 3, 319-325. MR 94m:35201. Zbl 0803.35098.

[27] _ On invariant measures for some infinite-dimensional dynamical systems, Ann. Inst. H. Poincaré Phys. Théor. 62 (1995), no. 3, 267-287. MR 96g:58116. Zbl 0826.35118.

[28] _ Invariant measures for infinite-dimensional dynamical systems with applications to a nonlinear Schrödinger equation, Algebraic and Geometric Methods in Mathematical Physics (Kaciveli, 1993), Math. Phys. Stud., vol. 19, Kluwer Acad. Publ., Dordrecht, 1996, pp. 471-476. MR 97b:35186. Zbl 0866.58043. 
[29] _ Invariant measures for the Korteweg-de Vries equation that are generated by higher conservation laws, Mat. Sb. 187 (1996), no. 6, 21-40 (Russian), [translated in Sb. Math. 187 (1996), no. 6, 803-822. Zbl 0926.35136]. MR 97h:35203.

[30] _ Invariant measures for the nonlinear Schrödinger equation, Proc. Int. Conf. dedicated to the 175th Anniversary of Professor P. L. Chebyshev's Birth (Moscow), Moscow State Univ. Publ., 1996, pp. 165-167 (Russian).

Peter E. Zhidkov: Bogoliubov Laboratory of Theoretical Physics, Joint Institute FOR NUCLEAR RESEARCH, 141980 DUBNA (MOSCOW REgION), RUSSIA

E-mail address: zhidkov@thsun1.jinr.ru 


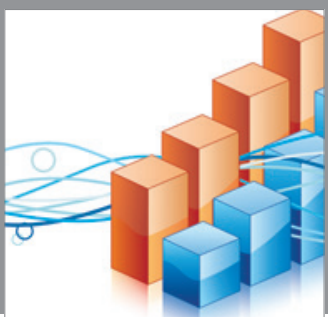

Advances in

Operations Research

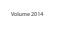

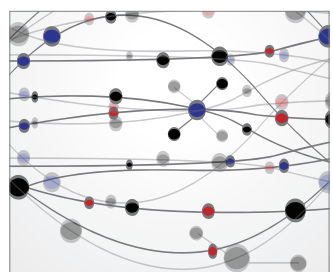

\section{The Scientific} World Journal
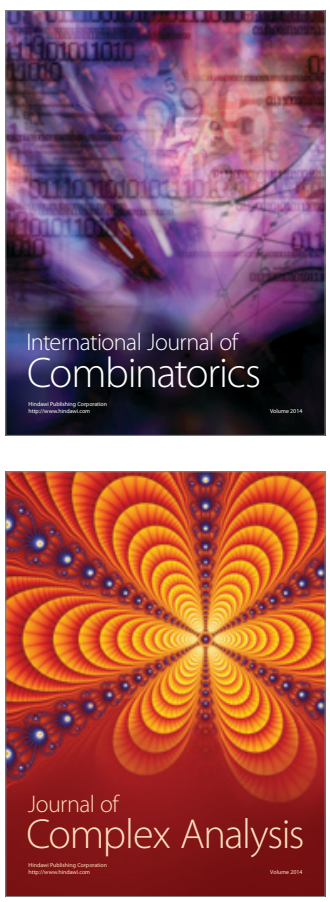

International Journal of

Mathematics and

Mathematical

Sciences
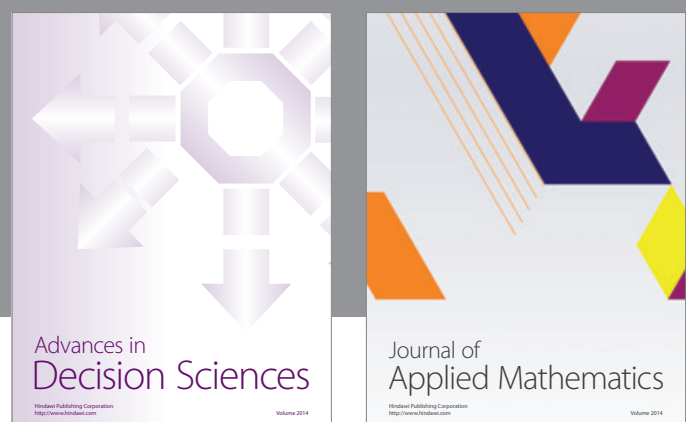

Journal of

Applied Mathematics
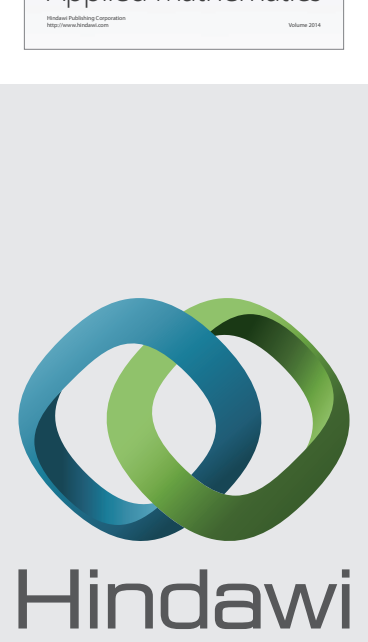

Submit your manuscripts at http://www.hindawi.com
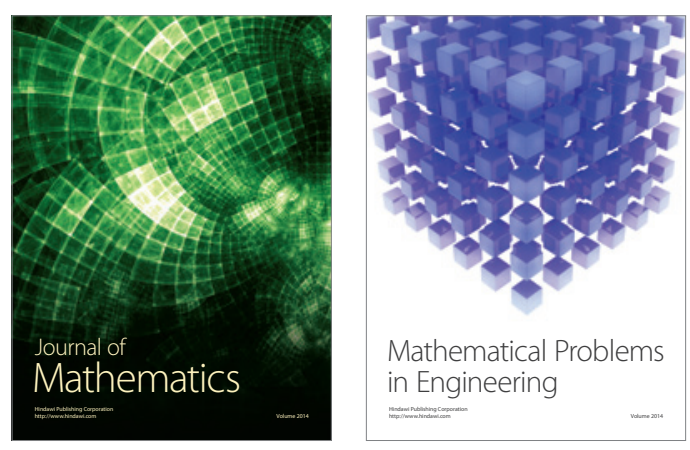

Mathematical Problems in Engineering
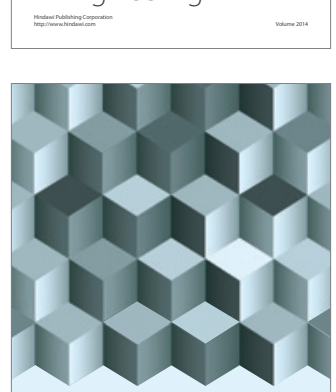

Journal of

Function Spaces
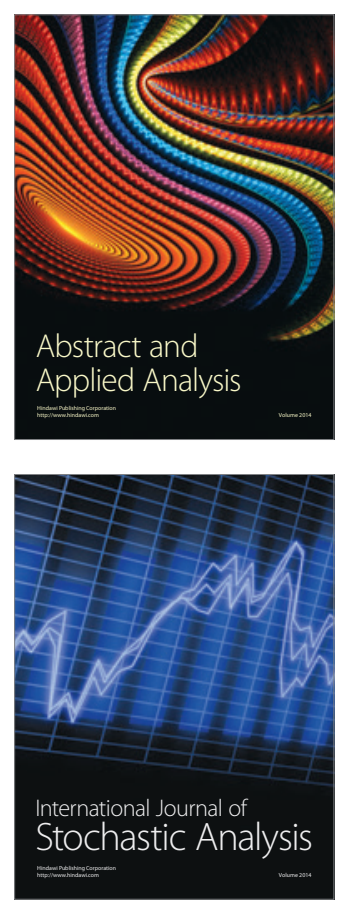

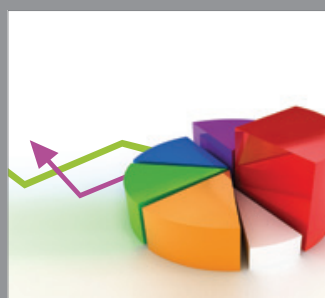

ournal of

Probability and Statistics

Promensencen
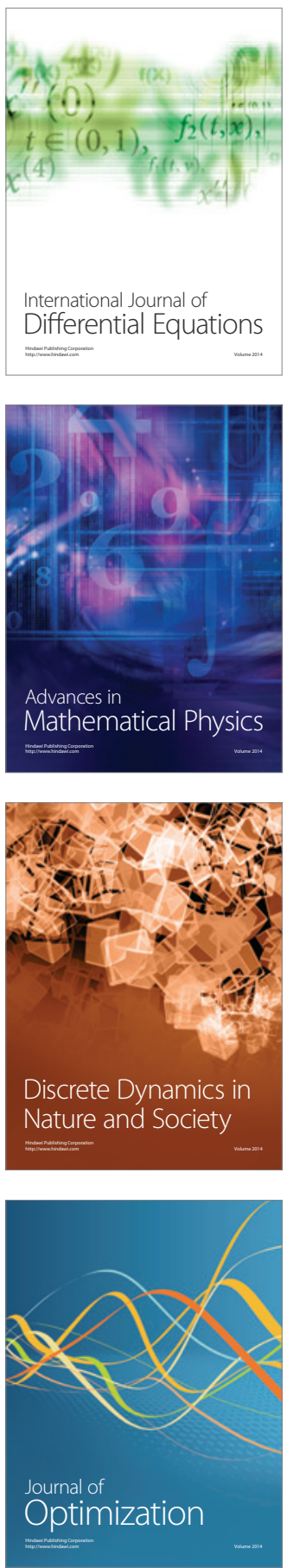